Pacific Journal of Mathematics

THE GROUP OF UNITS OF A COMMUTATIVE SEMIGROUP
RING

Robert William Gilder, JR. AND RAymond Hetman 


\title{
THE GROUP OF UNITS OF A COMMUTATIVE SEMIGROUP RING
}

\author{
Robert Gilmer and Raymond C. Heitmann
}

We seek a characterization, in terms of the coefficients and support of an element, of the units of the semigroup ring $R[X ; S]$, where $R$ is a commutative ring with identity and $S$ is an additive abelian semigroup with identity. Such a characterization requires some restrictions on the semigroup $S$.

We obtain results of the desired form in $\S 2$ for the case where $S$ is torsion-free and cancellative, and in $\S 3$ under the weaker hypothesis that $S$ is torsion-free and has no nonzero idempotents. Under this weaker hypothesis on $S$, the torsion subgroup of the group of units of $R[X ; S]$ is determined in $\S 4$ of this paper.

1. Introduction. If $R$ is a commutative ring with identity, then necessary and sufficient conditions are known in order that a polynomial $f \in R\left[\left\{X_{\lambda}\right\}\right]$ should be a unit. Namely, $f$ is a unit of $R\left[\left\{X_{\lambda}\right\}\right]$ if and only if the constant term of $f$ is a unit of $R$ and each other coefficient of $f$ is nilpotent $[10, p .683]$. In this paper we extend the preceding results by considering the group of units of the semigroup ring $R[X ; S]$, where $S$ is a torsion-free additive abelian semigroup with zero (the polynomial ring $R\left[\left\{X_{\lambda}\right\}_{\lambda \in A}\right]$ is isomorphic to the semigroup ring $R\left[X ; \sum_{i \in \Lambda} \oplus Z_{\lambda}\right]$, where each $Z_{\lambda}$ is the additive semigroup of nonnegative integers). Our main results concerning units of $R[X: S]$ are Theorems 2.4 and 3.2. Before stating these results, we indicate some conventions, terminology, and notation.

All rings considered are assumed to be commutative and to contain an identity element. Semigroups are assumed to be commutative, and we write the semigroup operation as addition; to indicate this, we frequently write "let $(S,+)$ be a semigroup". A semigroup with identity is called a monoid. Most of the semigroups we deal with are assumed to be monoids. If $R$ is a ring and $S$ is a semigroup, then we follow the notation of Northcott in [6, p. 128] in writing $R[X ; S]$ for the semigroup ring of $S$ over $R$ and in considering the elements of $R$ as 'polynomials' $r_{1} X^{s_{1}}+r_{2} X^{s_{2}}+\cdots+r_{n} X^{s_{n}}$ in $X$ with coefficients in $R$ and exponents in $S$. If $f$ is a nonzero element of $R[X ; S]$, then a representation of the preceding form, where $s_{1}, \cdots, s_{n}$ are distinct and each $r_{i}$ is nonzero, is called the canonical form of $f$, and $\left\{s_{i}\right\}_{i=1}^{n}$ is called the support of $f$. A unit of $R[X ; S]$ with only one element in its support is called a trivial unit; such a 
unit is necessarily of the form $r X^{s}$, where $r$ is a unit of $R$ and $s$ is an invertible element of $S$. The kind of characterization of units $f$ of $R[X ; S]$ that we seek is one stated in terms of the coefficients and the support of $f$. An examination of commutative integral group rings $Z G$ is sufficient to show that such a characterization cannot be expected unless restrictions are imposed on $S$. The primary restriction of this paper is that $S$ should be torsion-free, which is defined to mean that if $x$ and $y$ are distinct element of $S$, then $n x \neq n y$ for each positive integer $n$. Other restrictions imposed on $S$ on occasion are that $S$ be cancellative or that $S$ is without torsion, where cancellative is defined by the condition that $a+x=a+y$ implies that $x=y$ for all $a, x, y \in S$, and without torsion means that 0 is the only element $s$ of $S$ for which there exist distinct positive integers $m$ and $n$ such that $m s=n s$ (thus, torsion-free and without torsion are dual concepts). Proposition 3.1 show that a torsion-free monoid $S$ is without torsion if and only if 0 is the only idempotent of $S$; consequently, a torsion-free cancellative monoid is without torsion.

In $\S 2$, we assume that $S$ is a torsion-free cancellative monoid. Theorem 2.4 states that an element $f=\sum_{i=1}^{n} a_{i} X^{s_{i}}$ of $R[X ; S]$ is a unit if and only if there exists a positive integer $k$ such that $R=$ $\left(a_{1}^{k}\right) \oplus \cdots \oplus\left(a_{n}^{k}\right)$, and $s_{i}$ is invertible in $S$ for each $i$ such that $a_{i}$ is not nilpotent. It follows from Theorem 2.4 that $R[X ; S]$ admits only trivial units if and only if $R$ is reduced and either (1) $R$ is indecomposable, or (2) 0 is the only invertible element of $S$.

In $\S 3$, we replace the hypothesis that $S$ is cancellative by the weaker condition that 0 is the only idempotent of $S$. Theorem 3.2 yields the following characterization of units of $R[X ; S]$ in this less restrictive case. Assume that $(S,+)$ is a torison-free monoid containing no nonzero idempotents, and let $G$ be the set of invertible elements of $S$. Let $f=f_{1}+f_{2} \in R[X ; S]$, where $\operatorname{Supp}\left(f_{1}\right) \subseteq G$ and $\operatorname{Supp}\left(f_{2}\right) \subseteq$ $S-G$. Then $f$ is a unit of $R[X ; S]$ if and only if $f_{1}$ is a unit of $R[X ; G]$ and each coefficient of $f_{2}$ is nilpotent. Based upon Theorem 3.2 , it is shown in Proposition 3.8 that the Jacobson radical of $R[X ; S]$ coincides with the nilradical in the case where $S$ is torsion-free and contains no nonzero idempotents.

Section 4 of the paper is concerned with the problem of determining the torsion subgroup of the group of units of $R[X ; S]$, where $S$ is torsion-free and contains no nonzero idempotents. Theorem 4.7 is a composite of Theorems 4.4 and 4.6; it states that a unit $f$ of $R[X ; S]$ has finite order if and only if $0 \in \operatorname{Supp}(f)$, the coefficient of $X^{0}$ in $f$ is a unit of $R$ of finite order, and each other coefficient of $f$ is nilpotent of finite additive order.

2. The case where $S$ is torsion-free and cancellative. For a 
ring $R$ and a commutative monoid $(S,+)$, we resolve in this section the problem of characterizing the units of the semigroup ring $R[X ; S]$, where $S$ is torsion-free and cancellative. One version of the characterization is contained in an alternate form of Theorem 2.4: The element $f=a_{1} X^{s_{1}}+\cdots+a_{n} X^{s_{n}}$ of $R[X ; S]$ is a unit if and only if $R=\left(a_{1}, \cdots, a_{n}\right), a_{i} a_{j}$ is nilpotent for $i \neq j$, and $s_{i}$ is invertible in $S$ for each $i$ such that $a_{i}$ is not nilpotent.

Before proving our first result, Theorem 2.3, we record two results from [2, pp. 70,71] (see also [8], [9]).

Proposition 2.1. If $R$ is an integral domain, if $S$ is a torsionfree cancellative monoid, and if $f$ and $g$ are elements of $R[X ; S]$ such that $f g$ is a nonzero monomial, then $f$ and $g$ are monomials.

Corollary 2.2. Under the hypothesis of Proposition 2.1, $\left\{u X^{s} \mid u\right.$ is a unit of $R$ and $s$ is invertible in $S$ \} is the group of units of $R[X ; S]$. That is, $R[X ; S]$ has only trivial units.

Theorem 2.3 treats units of $R[X ; S]$ in the case where $R$ is a reduced ring - that is, a ring with nilradical (0).

THEOREM 2.3. Assume that $R$ is a reduced ring, that $S$ is a torsion-free cancellative monoid, and that $f=a_{1} X^{s_{1}}+\cdots+a_{n} X^{s_{n}}$ is the canonical form of the nonzero element $f$ of $R[X ; S]$. Then $f$ is a unit of $R[X ; S]$ if and only if each $s_{i}$ is invertible in $S$ and $R=$ $\left(a_{1}\right) \oplus \cdots \oplus\left(a_{n}\right)$.

Proof. Assume that each $s_{i}$ is invertible in $S$ and that $R=$ $\left(a_{1}\right) \oplus \cdots \oplus\left(a_{n}\right)$. Let $R_{i}=\left(a_{i}\right)$. Then $R[X ; S]=R_{1}[X ; S] \oplus \cdots \oplus$ $R_{n}[X ; S]$, and $f$ is a unit of $R[X ; S]$ if and only if $f R_{i}[X ; S]=$ $R_{i}[X ; S]$ for each $i$. But $f R_{i}[X ; S]=a_{i} X^{s_{i}} R_{i}[X ; S]$ and $a_{i} X^{s_{i}}$ is a unit of $R_{i}[X ; S]$ since $a_{i}$ is a unit of $R_{i}=\left(a_{i}\right)$ and $s_{i}$ is invertible in $S$. Consequently, $f$ is a unit of $R[X ; S]$.

We assume, conversely, that $f$ is a unit of $R[X ; S]$. If $P$ is a proper prime ideal of $R$, then the natural homomorphism $R \rightarrow R / P$ of $R$ onto $R / P$ has a unique extension to a homomorphism

$$
\phi_{P}: R[X ; S] \longrightarrow(R / P)[X ; S]
$$

such that $\phi_{P}\left(X^{s}\right)=X^{s}$ for each $s$ in $S$. Since $\phi_{P}(f)$ is a unit of $(R / P)[X ; S]$ for each $P$, Corollary 2.2 implies that $\phi_{P}(f)$ is of the form $u X^{s}$, where $u$ is invertible in $R / P$ and $s$ is invertible in $S$. To prove then that each $s_{i}$ is invertible in $S$, note that since $a_{i}$ is nonzero, $a_{i}$ is not nilpotent, and consequently, $a_{i} \notin P$ for some proper prime ideal $P$ of $R$. It follows from the definition of $\phi_{P}$ that $\phi_{P}(f)=$ 
$\phi_{P}\left(a_{i}\right) X^{s_{i}}$ so that $s_{i}$ is invertible, as asserted. If $Q$ is a proper prime ideal of $R$, then since $\phi_{Q}(f)$ is a nonzero monomial, one and only one of the coefficients $a_{1}, a_{2}, \cdots, a_{n}$ is not in $Q$. This implies:

(1) $\left(a_{1}, \cdots, a_{n}\right)=R$.

(2) For $i \neq j, a_{i} a_{j}$ is in each proper prime ideal of $R$, and hence $a_{i} a_{j}$ is nilpotent. Since $R$ has nilradical (0), it follows that $a_{i} a_{j}=0$.

(3) $\quad R=\left(a_{1}\right) \oplus\left(a_{2}\right) \oplus \cdots \oplus\left(a_{n}\right)$, for (1) implies that $R=\left(a_{1}\right)+$ $\cdots+\left(a_{n}\right)$ and thus, for example, $\left(a_{1}\right) \cap\left(a_{2}, \cdots, a_{n}\right)=\left(a_{1} a_{2}, \cdots, a_{1} a_{n}\right)=$ (0).

In passing from Theorem 2.3 to the case where $R$ need not be reduced, we use the fact that if $x \in R$ and if $A$ is an ideal of $R$ contained in the Jacobson radical of $R$, then $x$ is a unit of $R$ if and only if $x+A$ is a unit of $R / A$.

THEOREM 2.4. Assume that $S$ is a torsion-free cancellative monoid. If $f=a_{1} X^{s_{1}}+\cdots+a_{n} X^{s_{n}}$ is the canonical form of $f$, then $f$ is a unit of $R[X ; S]$ if and only if there exists a positive integer $k$ such that $R=\left(a_{1}^{k}\right) \oplus \cdots \oplus\left(a_{n}^{k}\right)$, and $s_{i}$ is invertible in $S$ for each $i$ such that $a_{i}$ is not nilpotent.

Proof. If $N$ is the nilradical of $R$ and if $\phi_{N}: R[X] \rightarrow(R / N)[X]$ is the homomorphism induced, as in the proof of Theorem 2.3, by the canonical projection of $R$ onto $R / N$, then $f$ is a unit of $R[X ; S]$ if and only if $\phi_{N}(f)$ is a unit of $(R / N)[X ; S]$. By Theorem 2.3, $\phi_{N}(f)$ is a unit of $(R / N)[X ; S]$ if and only if $R / N=\left(a_{1}+N\right) \oplus \cdots \oplus\left(a_{n}+N\right)$ and $s_{i}$ is invertible for each $s_{i} \in \operatorname{Supp}\left(\phi_{N}(f)\right)$, that is, for each $i$ such that $a_{i}$ is not nilpotent. Hence, Theorem 2.4 will be proved if we can show that $R / N=\left(a_{1}+N\right) \oplus \cdots \oplus\left(a_{n}+N\right)$ if and only if there exists a positive integer $k$ such that $R=\left(a_{1}^{k}\right) \oplus \cdots \oplus\left(a_{n}^{k}\right)$.

If $R / N=\left(a_{1}+N\right) \oplus \cdots \oplus\left(a_{n}+N\right)$, then $R=\left(a_{1}, \cdots, a_{n}\right)+N=$ $\left(a_{1}, \cdots, a_{n}\right)=\left(a_{1}^{k}, \cdots, a_{n}^{k}\right)$ for every $k$. Because the sum is direct, it follows that $\left(a_{i}+N\right)\left(a_{j}+N\right)=N$ for $i \neq j$, and hence $a_{i} a_{j}$ is nilpotent for $i \neq j$. Choose $k$ sufficiently large so that $\left(a_{i} a_{j}\right)^{k}=0$ for all pairs $i \neq j$. Since $a_{i}^{k} a_{j}^{k}=0$ for $i \neq j$, it follows as in the proof of Theorem 2.3 that the sum $R=\left(a_{1}^{k}\right)+\cdots+\left(a_{n}^{k}\right)$ is direct. Conversely, if $R=$ $\left(a_{1}^{k}\right) \oplus \cdots \oplus\left(a_{n}^{k}\right)$, then obviously $R / N=\left(a_{1}+N\right)+\cdots+\left(a_{n}+N\right)$. As before, to prove that this sum is direct, it suffices to show that $\left(a_{i}+N\right)\left(a_{j}+N\right)=N$ - that is, $a_{i} a_{j}$ is nilpotent for $i \neq j$. Since $a_{i}^{k} a_{j}^{k}=0$, this follows immediately. Therefore the equivalence has been established, and this completes the proof of Theorem 2.4.

Note that the proof of Theorem 2.4 shows that the condition that $R=\left(a_{1}^{k}\right) \oplus \cdots \oplus\left(a_{n}^{k}\right)$ for some positive integer $k$ is equivalent to the assertion that $R=\left(a_{1}, \cdots, a_{n}\right)$ and $a_{i} a_{j}$ is nilpotent for $i \neq j$. 
Corollary 2.5. Assume that $G$ is a torsion-free group and $f=\sum_{i=1}^{n} a_{i} X^{g_{i}} \in R[X ; G]$. The following conditions are equivalent.

(1) $f$ is a unit of $R[X ; G]$.

(2) $R=\left(a_{1}, a_{2}, \cdots, a_{n}\right)$ and $a_{i} a_{j}$ is nilpotent for $i \neq j$.

(3) There exists a positive integer k such that $R=\left(a_{1}^{k}\right) \oplus \cdots \oplus$ $\left(a_{n}^{k}\right)$.

Using Theorem 2.4, we are able to give necessary and sufficient conditions in order that the semigroup ring $R[X ; S]$, where $S$ is torsion-free and cancellative, should have only trivial units.

THEOREM 2.6. Assume that $S$ is a nonzero torsion-free cancellative monoid and that $G$ is the group of invertible elements of $S$. The semigroup ring $R[X ; S]$ has only trivial units if and only if $R$ is reduced and either (1) or (2) is satisfied:

(1) $R$ is indecomposable.

(2) $G=\{0\}$.

Proof. It is immediate from Theorem 2.4 that $R[X ; S]$ has only trivial units if $R$ is reduced and (1) or (2) is satisfied. If $R$ is not reduced and if $r$ is a nonzero nilpotent of $R$, then $1+r X^{s}$ is a nontrivial unit of $R[X ; S]$ for each nonzero element $s$ of $S$. On the other hand, if $R$ is decomposable and $G \neq\{0\}$, then take a decomposition $1=e_{1}+e_{2}$ into nonzero orthogonal idempotents of $R$ and consider a nonzero element $g$ of $G$. Then $e_{1}+e_{2} X^{g}$ is a nontrivial unit of $R[X ; S]$ with inverse $e_{1}+e_{2} X^{-g}$. This completes the proof of Theorem 2.6.

At first glance it may not be clear that the hypotheses that $S$ is torsion-free and cancellative are used in an essential way in the proofs of Theorems 2.3 and 2.4. Nevertheless, the assumptions concerning $S$ enter our considerations through Corollary 2.2, which depends essentially on the fact that $S$ can be ordered if $S$ is torsionfree and cancellative. In the next section we are able to prove some substantial results about units of $R[X ; S]$ without assuming that $S$ is cancellative. The case where $S$ is a group already shows that a result like Theorem 2.4 cannot be expected if $S$ is assumed to be cancellative, but not torsion-free. It is well-known, for example, that the integral group ring $Z G$ has nontrivial units for each finite abelian group $G$ of exponent not dividing 4 or 6 [4].

3. The case where $S$ is torsion-free and without torsion. We begin this section by taking another look at Theorem 2.4, in light of the following considerations. If $(S,+)$ is a monoid, recall that an ideal of $S$ is a nonempty subset $I$ of $S$ such that $s+I \subseteq I$ for 
each $s \in S$, and that the ideal $I$ is prime if $x+y \in I$ implies that $x \in I$ or $y \in I$ for all $x, y \in S$. Assume that $I$ is a proper prime ideal of $S$. Then $S=(S-I) \cup I$, where $S-I$ is a submonoid of $S$, and this partition of $S$ induces the decomposition $R[X ; S]=R[X ; S-I]+$ $R[X ; I]$ of the semigroup ring, where $R[X ; S-I]$ is a subring, and $R[X ; I]$ is an ideal, of $R[X ; S]$. Moreover, since $R[X ; S-I] \cap$ $R[X ; I]=(0)$, the sum $R[X ; S-I]+R[X ; I]$ is direct in the grouptheoretic sense. If $f \in R[X ; S]$ and if $f=f_{1}+f_{2}$, where $f_{1} \in R[X ; S-I]$ and $f_{2} \in R[X ; I]$, then $f_{1}$ is called the $(S-I)$-component of $f$ and $f_{2}$ is the I-component of $f$. Let $f, g \in R[X ; S]$ and let $f=f_{1}+f_{2}, g=$ $g_{1}+g_{2}$, where $f_{1}, g_{1} \in R[X ; S-I]$ and $f_{2}, g_{2} \in R[X ; I]$. Then $f g=$ $f_{1} g_{1}+\left(f_{1} g_{2}+f_{2} g_{1}+f_{2} g_{2}\right)$ is the corresponding decomposition of the product $f g$. In particular, $f g=1$ if and only if $f_{1} g_{1}=1$ and $f_{1} g_{2}+$ $f_{2} g_{1}+f_{2} g_{2}=0$. Thus $f_{1}$ is a unit of $R[X ; S-I]$ if $f$ is a unit of $R[X ; S]$. Let $G$ be the set of invertible elements of $S$. If $S \neq G$, then $I=S-G$ is a prime ideal of $S$, and we obtain the decomposition $R[X ; S]=R[X ; G]+R[X ; S-G]$. Although the empty set is not considered to be an ideal of $S$, we find it convenient to consider the decomposition $R[X ; S]=R[X ; G]+(0)$ if $G=S$; in this case, the $G$-component and $(S-G)$-component of $f$ are taken to be $f$ and 0 , respectively. In terms of the decomposition of $R[X ; S]$ induced by $G$, Theorem 2.4 can be interpreted as follows.

(*) Assume that $G$ is the set of invertible elements of the monoid $(S,+)$. Consider $f \in R[X ; S]$, and let $f_{1}$ and $f_{2}$, respectively, be the G-component and $(S-G)$-component of $f$. If $S$ is torsion-free and cancellative, then $f$ is a unit of $R[X ; S]$ if and only if $f_{1}$ is a unit of $R[X ; G]$ and $f_{2} \in N[X ; S-G]$, where $N$ is the nilradical of $R$.

In the statement of $\left(^{*}\right)$, we remark that $N[X ; S-G]$ is taken to be (0) in the case where $S=G$. Note that the following assertions in $\left(^{*}\right)$ are valid without the hypothesis that $S$ is torsion-free and cancellative: (1) If $f$ is a unit of $R[X ; S]$, then $f_{1}$ is a unit of $R[X ; G]$. (2) If $f_{1}$ is a unit of $R[X ; G]$ and if each coefficient of $f_{2}$ is nilpotent, then $f$ is a unit of $R[X ; S]$. Because of (2), it is natural to attempt to prove $\left({ }^{*}\right)$ under weakened hypotheses on $S$. Also, we are interested in obtaining a result like Theorem 2.4 in the case where $S$ is not assumed to be cancellative. Each of these goals is realized in Theorem 3.2 , which shows that $\left(^{*}\right)$ is valid if $S$ is torsion-free and without torsion. The terminology here is as follows. An element $s$ of a semigroup $S_{0}$ is a torsion element if there exist distinct positive integers $m$ and $n$ such that $m s=n s$. A monoid $S$ is said to be without torsion if 0 is the only torsion element of $S$. A torsion-free cancellative monoid is without torsion; the next result gives conditions 
under which an arbitrary torsion-free monoid is without torsion.

Proposition 3.1. Let $(S,+)$ be a torsion-free monoid. Then $S$ is without torsion if and only if 0 is the only idempotent element of $S$.

Proof. If $S$ is without torsion, then it is clear that 0 is the only idempotent element of $S$. Conversely, assume that 0 is the only idempotent of $S$ and let $x$ be a torsion element of $S$. Then $\langle x\rangle$, the subsemigroup of $S$ generated by $x$, contains an idempotent $k x[1$, $\S 1.6]$. Thus $k x=0$ by assumption, and since $S$ is torsion-free, the equality $k x=k \cdot 0$ implies that $x=0$. Hence $S$ is without torsion.

In view of Proposition 3.1, we choose henceforth to speak of a torsion-free monoid containing no nonzero idempotents, rather than a torsion-free monoid without torsion.

We observe at this point that the conclusion of $\left(^{*}\right)$ need not extend to the case where $S$ contains a nonzero idempotent $s$. For example, the element $u=1-2 X^{s}$ of the integral semigroup ring $Z[X ; S]$ is a unit since $u^{2}=1$, but the $(S-G)$-component of $u$ is $-2 X^{s}$, and -2 is not nilpotent in $Z$.

THEOREM 3.2. Assume that $(S,+)$ is a torsion-free monoid containing no nonzero idempotents. Let $G$ be the set of invertible elements of $S$ and let $N$ be the nilradical of the ring $R$. An element $f$ of $R[X ; S]$ is a unit if and only if the G-component of $f$ is a unit of $R[X ; G]$ and the $(S-G)$-component of $f$ is in $N[X ; S-G]$.

The proof of Theorem 3.2 will be given following a sequence of lemmas, each of which is a special case of Theorem 3.2 itself.

LEMMA 3.3. Let $R$ be a field of characteristic $p \neq 0$, and let $(S,+)$ be a torsion-free monoid containing no nonzero idempotent element. Assume that $S^{*}=S-\{0\}$ is the only proper prime ideal of $S$. If $1+f$ is a unit of $R[X ; S]$, where $f \in R\left[X ; S^{*}\right]$, then $f=0$.

Proof. We first claim that the set $I=\left\{s \in S \mid\right.$ there exists $t \in S^{*}$ such that $s=s+t\}$ is empty. If not, there exists $t \in S^{*}$ such that the set $I_{t}=\{s \in S \mid t+s=s\}$ is also nonempty. The set $I_{t}$ is a proper ideal of $S$, and since $S^{*}$ is the unique proper prime ideal of $S$, it follows that $I_{t}$ meets the subsemigroup $\langle t\rangle=\{k t\}_{k=1}^{\infty}$ of $S$ [3, Lemma 4]. Thus $t+m t=m t$ for some positive integer $m$, and since $t \neq 0$, this contradicts Proposition 3.1. Consequently, $I$ is empty.

We denote by $\sim$ the cancellation congruence of $S$ - that is, $a \sim b$ 
if and only if there exists an element $c \in S$ such that $a+c=b+c$. Let $\bar{S}=S / \sim$ denote the factor monoid of $S$ with respect to $\sim$, and for $s \in S$, let [s] be the class of $s$ with respect to $\sim$. It is well-known that $\bar{S}$ is cancellative. We proceed to prove that $\bar{S}$ is torsion-free. If $n[a]=n[b]$, then $n a \sim n b$ and $n a+t=n b+t$ for some $t \in S$. It follows that $n(a+t)=n(b+t)$, and since $S$ is torsion-free, $a+t=$ $b+t$. Hence $a \sim b,[a]=[b]$, and $\bar{S}$ is torsion-free. Let $[x]$ be an invertible element of $\bar{S}$, say $[0]=[x]+[y]=[x+y]$. Then $x+y \sim 0$, and since $I$ is empty, we conclude that $x+y=0$. Because $S^{*}$ is the only proper prime ideal of $S$, it follows that 0 is the only invertible element of $S$. Therefore $x=y=0$, and [0] is the only invertible element of $\bar{S}$. Summarizing, $\bar{S}$ is a torsion-free cancellative monoid with no invertible element other than [0]. It then follows from Theorem 2.6 that the only units of $R[X ; \bar{S}]$ are those of $R$.

The natural projection of $S$ onto $\bar{S}$ can be uniquely extended to an $R$-homomorphism $\theta$ of $R[X ; S]$ onto $R[X ; \bar{S}]$. Thus $\theta(1+f)=$ $1+\theta(f)$ is a unit of $R[X ; \bar{S}]$, and since $I$ is empty, it follows that [0] is not in the support of $\theta(f)$. Consequently, $\theta(f)=0$. Let $f=$ $r_{1} X^{s_{1}}+\cdots+r_{n} X^{s_{n}}$. If $s_{i}, s_{j} \in \operatorname{Supp}(f)$ and if $s_{i} \sim s_{j}$, then we choose $t_{i j} \in S$ such that $s_{i}+t_{i j}=s_{j}+t_{i j}$. Let $t=\sum_{i \sim j} t_{i j}$, where the symbol $\sum_{i \sim j}$ indicates that the sum is taken over all pairs $(i, j)$ with $s_{i}, s_{j} \in$ $\operatorname{Supp}(f)$ and $s_{i} \sim s_{j}$. We note that

$$
s_{i}+t \sim s_{j}+t \Longleftrightarrow s_{i} \sim s_{j} \Longleftrightarrow s_{i}+t=s_{j}+t \text {. }
$$

Next consider $X^{t} f=r_{1} X^{s_{1}+t}+\cdots+r_{n} X^{s_{n}+t}=r_{1}^{\prime} X^{s_{k}^{\prime}}+\cdots+r_{k}^{\prime} X^{s_{k}^{\prime}}$, where $s_{1}^{\prime}, \cdots, s_{k}^{\prime}$ are distinct. We have $0=\theta\left(X^{t}\right) \theta(f)=\theta\left(X^{t} f\right)=r_{1}^{\prime} X^{\left[s_{k}^{\prime}\right]}+$ $\cdots+r_{k}^{\prime} Y^{\left[s_{k}^{\prime}\right]}$, and since $s_{i}^{\prime} \nsim s_{j}^{\prime}$ for $i \neq j$, it follows that $r_{1}^{\prime}=\cdots=$ $r_{k}^{\prime}=0$. Therefore $X^{t} f=0$. In particular, if $t=0$, then $f=0$ and the proof is complete.

Assume that $t \neq 0$. Because $1+f$ is a unit, it has an inverse $(1+f)^{-1}$, which is easily seen to be of the form $1+g$, where $0 \notin \operatorname{Supp}(g)$. Again using the fact that $S^{*}$ is the only proper prime ideal of $S$, we see that a positive integral multiple of each element of $\operatorname{Supp}(g)$ is in in the ideal $t+S$ of $S$. Choose $p^{k}$ large enough (recall that $p$ is the characteristic of $R$ ) so that $p^{k} u \in t+S$ for each $u \in \operatorname{Supp}(g)$. Since $1=(1+f)(1+g)$, we have $0=f+g+$ $f g=(f+g+f g)^{p^{k}}=f^{p^{k}}+g^{p^{k}}+f^{p^{k}} g^{p^{k}}$. By choice of $k, X^{t}$ is a factor of $g^{p^{k}}$, and hence $f^{p^{k}} g^{p^{k}}=0$. Consequently, $f^{p^{k}}=-g^{p^{k}}$ and $0=f^{p^{k}} g^{p^{k}}=-f^{2 p^{k}}$. Hence $f=0$, for Corollary 2.3 of [7] implies that the ring $R[X ; S]$ is reduced.

The statement of Lemma 3.4 is obtained merely by weakening the hypothesis in Lemma 3.3 that $S-\{0\}$ is the only proper prime of $S$ to the assumption that 0 is the only invertible element of $S$. 
LEMMA 3.4. Let $R$ be a field of characteristic $p \neq 0$, and let $(S,+)$ be a torsion-free monoid containing no nonzero idempotent element. Assume that 0 is the only invertible element of $S$. If $1+f$ is a unit of $R[X ; S]$, where $f \in R[X ; S-\{0\}]$, then $f=0$.

Proof. Assume that Lemma 3.4 is false. We write $(1+f)^{-1}=$ $1+g$, where $0 \notin \operatorname{Supp}(g)$. We may assume that $|\operatorname{Supp}(f)|+|\operatorname{Supp}(g)|$ is minimal among all counterexamples to the statement of the lemma. Replacing $S$ by the submonoid of $S$ generated by $U=\operatorname{Supp}(f) U$ $\operatorname{Supp}(g)$, we also assume, without loss of generality, that the subsemigroup of $S$ generated by $U$ is $S^{*}=S-\{0\}$. Because 0 is the only invertible element of $S$, it follows that $S^{*}$ is a prime ideal of $S$. In light of Lemma 3.3, we conclude that there exists a prime ideal $P$ of $S$ properly contained in $S^{*}$.

As $S^{*}=\left(S^{*}-P\right) \cup P$ and $S^{*}$ is generated by $U$, it is clear that $U \nsubseteq P$ and $U \nsubseteq\left(S^{*}-P\right)$. We write $1+f=1+f_{1}+f_{2}$, where $1+f_{1}$ is the $(S-P)$-component of $1+f$ and $f_{2}$ is the $P$-component of $f$; similarly, $1+g=1+g_{1}+g_{2}$, where $1+g_{1}$ is the $(S-P)$-component of $1+g$. It follows from previous observations that $1+f_{1}$ is a unit of the semigroup ring $R[X ; S-P]$ and that $1+g_{1}$ is the inverse of $1+f_{1}$. Since $U \nsubseteq S^{*}-P$, either $f_{2}$ or $g_{2}$ is nonzero, and $\left|\operatorname{Supp}\left(f_{1}\right)\right|+$ $\left|\operatorname{Supp}\left(g_{1}\right)\right|<|\operatorname{Supp}(f)|+|\operatorname{Supp}(g)|$. Moreover, as $U \nsubseteq P$, either $f_{1}$ or $g_{1}$ is nonzero. Thus $1+f_{1}$ and $1+g_{1}$ provide a counterexample to Lemma 3.4 that contradicts the assumption that $|\operatorname{Supp} f|+|\operatorname{Supp} g|$ is minimal. This completes the proof of Lemma 3.4.

LEMMA 3.5. Let $R$ be a field of characteristic $p \neq 0$, and let $(S,+)$ be a torsion-free monoid containing no nonzero idempotent elements. Each unit of $R[X ; S]$ is in $R[X ; G]$, where $G$ is the set of invertible elements of $S$.

Proof. Let $u$ be a unit of $R[X ; S]$ with inverse $v$ and write $u=u_{1}+u_{2}, v=v_{1}+v_{2}$, where $u_{1}$ and $v_{1}$ are the G-components of $u$ and $v$, respectively. Then $u_{1}$ is a unit of $R[X ; G]$ with inverse $v_{1}$, and hence $v_{1} u=1+v_{1} u_{2}$ is a unit of $R[X ; S]$ with inverse $u_{1} v=$ $1+u_{1} v_{2}$. As $v_{1} u_{2}$ and $u_{1} v_{2}$ are in $R[X ; S-G]$, it follows that $1+v_{1} u_{2}$ is, in fact, a unit of the semigroup ring $R[X ;(S-G) \cup\{0\}]$. Applying Lemma 3.4 in this perspective, we conclude that $v_{1} u_{2}=0$, and hence $u_{2}=0$. Consequently, $u=u_{1} \in R[X ; G]$, as was to be proved.

The next lemma is the final preliminary result needed for the proof of Theorem 3.2. The statement of Lemma 3.6 differs from that of Lemma 3.5 only in that $R$ is assumed to be a reduced ring, rather than a field of nonzero characteristic. 
LEMMA 3.6. Let $R$ be a reduced ring, and let $(S,+)$ be a torsionfree monoid containing no nonzero idempotent elements. Each unit of $R[X ; S]$ is in $R[X ; G]$, where $G$ is the set of invertible elements of $S$.

Proof. Let $f=f_{1} X^{s_{1}}+\cdots+f_{n} X^{s_{n}}$ be a unit of $R[X ; S]$, with inverse $g=g_{1} X^{t_{1}}+\cdots+g_{m} X^{t_{m}}$. Let $\Pi$ be the prime subring of $R$ and let $R_{0}=\Pi\left[f_{1}, \cdots, f_{n}, g_{1}, \cdots, g_{m}\right]$. The ring $R_{0}$ is reduced, and $R_{0}$ is known to be a Hilbert ring such that $R_{0} / M$ is a finite field for each maximal ideal $M$ of $R_{0}$ (see $\S 3$ of [7]). In particular, if $\left\{M_{\lambda}\right\}_{\lambda \in A}$ is the family of maximal ideals of $R_{0}$, then $\bigcap_{\lambda \in A} M_{\lambda}$ is the nilradical of $R_{0}$ (hence $\cap M_{\lambda}=(0)$ ) and each $R_{0} / M_{\lambda}$ is a field of nonzero characteristic. For $\lambda \in \Lambda$, denote by $\varphi_{\lambda}$ the canonical homomorphism of $R_{0}[X ; S]$ onto $\left(R_{0} / M_{\lambda}\right)[X ; S]$. Noting that $f$ is a unit of $R_{0}[X ; S]$, it follows that $\varphi_{\lambda}(f)$ is a unit of $\left(R_{0} / M_{\lambda}\right)[X ; S]$ for each $\lambda$. By Lemma $3.5, \varphi_{\lambda}(f) \in\left(R_{0} / M_{\lambda}\right)[X ; G]$. Thus if $h$ is the $(S-G)$-component of $f$, then $h \in \bigcap_{\lambda \in \Lambda}\left(M_{\lambda}[X ; S-G]\right)=\left(\bigcap_{\lambda \in \Lambda} M_{\lambda}\right)[X ; S-G]=(0)$. Therefore $f \in R[X ; G]$, as asserted.

Proof of Theorem 3.2. We have already observed that $f$ is a unit of $R[X ; S]$ if the G-component of $f$ is a unit of $R[X ; G]$ and the $(S-G)$-component of $f$ is in $N[X ; S-G]$. Conversely, assume that $f$ is a unit of $R[X ; S]$. If $\varphi$ is the canonical homomorphism of $R[X ; S]$ onto $(R / N)[X ; S]$, then $\varphi$ has kernel $N[X ; S]$. Since $\varphi(f)$ is a unit of $(R / N)[X ; S]$, where the ring $R / N$ is reduced, Lemma 3.6 shows that $\varphi(f) \in(R / N)[X ; G]$. Let $h$ be a preimage of $\varphi(f)$ in $R[X ; G]$, and let $g=f-h$. Since $\varphi(g)=0, g$ is in $N[X ; S]$, the kernel of $\varphi$. Therefore the $(S-G)$-component of $g$ is in $N[X ; S-G]$. Because $f=h+g$, where $h \in R[X$; G], it follows that $f$ and $g$ have the same $(S-G)$-component. Consequently, the $(S-G)$-component of $f$ is in $N[X ; S-G]$. Because we already know that the $G$ component of $f$ is a unit of $R[X ; G]$, this completes the proof of Theorem 3.2.

Using Theorem 3.2, it is easy to extend Theorem 2.6 to the case where the monoid $S$ is torsion-free and contains no nonzero idempotents. We state this result without proof.

THEOREM 3.7. Assume that $S$ is a nonzero torsion-free monoid containing no nonzero idempotents and that $G$ is the group of invertible elements of $S$. The semigroup ring $R[X ; S]$ has only trivial units if and only if $R$ is reduced and either (1) or (2) is satisfied:

(1) $R$ is indecomposable.

(2) $G=\{0\}$. 
Theorem 3.2 can also be used to determine the Jacobson radical of $R[X ; S]$ in the case where $S$ is torsion-free and contains no nonzero idempotents.

Proposition 3.8. If $S \neq\{0\}$ is torsion-free and contains no nonzero idempotents, then the Jacobson radical of $R[X ; S]$ is $N[X ; S]$, where $N$ is the nilradical of $R$. Thus, the nilradical and the Jacobson radical of $R[X ; S]$ coincide.

Proof. Let $f$ be an element of the Jacobson radical of $R[X ; S]$ and let $G$ be the set of invertible elements of $S$. Since $1+f$ is a unit of $R[X ; S]$, it follows from Theorem 3.2 that the $(S-G)$ component of $f$ is in $N[X ; S-G] \subseteq N[X ; S]$. Let $h$ be the $(S-G)$ component of $f$. If $f=h$, then the proof is complete. If $f \neq h$, then by replacing $f$ with $f-h$, we can assume without loss of generality that $f \in R[X ; G]$. We prove that $f \in N[X$; $G]$ by considering separately the cases $G=(0)$ and $G \neq(0)$. If $G=(0)$, then we choose a nonzero element $s \in S$. Since $f \in R$ and since $1+f X^{s}$ is a unit of $R[X ; S]$, Theorem 3.2 shows that $f \in N$ in this case. If $G \neq(0)$, then $G$ is infinite because $G$ is torsion-free. We can therefore choose $g \in G$ so that $0 \notin \operatorname{Supp}\left(X^{g} f\right)$. It follows from Theorem 3.2 that $1+X^{g} f$ is a unit of $R[X ; G]$, and then Corollary 2.5 shows that each coefficient of $f$ is nilpotent. That is, $f \in N[X ; G]$ and the proof of Proposition 3.8 is complete.

4. Units of finite order. Having determined in Theorem 3.2 and Corollary 2.5 the group of units of $R[X ; S]$ for $S$ torsion-free and containing no nonzero idempotents, it is natural to ask for conditions under which a unit $f$ of $R[X ; S]$ has finite order. Consistent with previous standards, we desire that such conditions be given in terms of the coefficients and support of $f$. Theorems 4.4, 4.6, and 4.7 of this section contain results in this direction that we consider to be satisfactory. Because of Theorem 3.2, there is a natural way of considering a unit of $R[X ; S]$ in the form $u+n$, where $u$ is a unit of $R[X ; G]$ and $n$ is nilpotent. The initial results of this section are concerned with the problem of determining conditions under which such a unit $u+n$ is of finite order.

Theorem 4.1. Assume that $n$ is a nilpotent element of $R$ and $u$ is a unit of $R$ of finite order. The unit $u+n$ has finite order if and only if $n$ has finite additive order.

Proof. It is well-known that $u+n$ is a unit of $R$. Since $u$ has finite order, $u+n$ has finite order if and only if $u^{-1}(u+n)=$ 
$1+u^{-1} n$ has finite order. Moreover, $u^{-1} n$ is nilpotent and $u^{-1} n$ has finite additive order if and only if $n$ has finite additive order. We therefore assume without loss of generality that $u=1$.

If $1+n$ has finite order $t$, then $1=(1+n)^{t}=1+t n+r n^{2}$ for some element $r \in R$. Therefore

$$
t n=-r n^{2}, t^{2} n=t\left(-r n^{2}\right)=(-r n)\left(-r n^{2}\right)=r^{2} n^{3},
$$

and by induction, it follows that $t^{k} n=(-r)^{k} n^{k+1}$ for each positive integer $k$. Let $m$ be the order of nilpotence of $n$. Then $t^{m-1} n=0$, and hence $n$ has finite additive order.

Conversely, assume that $k n=0$ for some positive integer $k$. We prove that $1+n$ has finite order by induction on the number of (not necessarily distinct) prime factors of $k$. If $k=p$ is prime, then choose the positive integer $m$ so that $n^{p^{m}}=0$. We have $(1+n)^{p^{m}}=$ 1 , so $1+n$ has finite order. If the result is assumed for integers with $h$ prime factors and if $k=p_{1} \cdots p_{h} p$ has $h+1$ prime factors, then we choose $m$ large enough so that $n^{p^{m}}=0$. We have $(1+n)^{p^{m}}=$ $1+p r n$ for some $r \in R$, and $(k / p) p n r=0$. By the induction hypothesis, $1+p r n$ has finite order. Consequently, $1+n$ also has finite order.

CoRollary 4.2. Assume that $u$ is a unit of $R$ and $n$ is a nilpotent element of $R$ of finite additive order. Then $u+n$ has finite order if and only if $u$ has finite order.

Proof. Theorem 4.1 shows that $u+n$ has finite order if $u$ has finite order. If $u+n$ has finite order, then since $-n$ is nilpotent of finite additive order, Theorem 4.1 shows that $u=u+n-n$ has finite order.

CoROLlaRY 4.3. If $R$ has nonzero characteristic, if $u$ is a unit of $R$, and if $n$ is a nilpotent element of $R$, then $u+n$ has finite order if and only if $u$ has finite order.

We remark that Corollary 4.3 fails without the hypothesis that $R$ has nonzero characteristic. For example, let $R=Q[X] /\left(X^{2}\right)=Q[x]$. Then $x$ is nilpotent and does not have finite additive order. Therefore $1+x$ is a unit that is not of finite order. But $(1+x)-x=1$ is of finite order.

THeOREM 4.4. Assume that $(S,+)$ is a torsion-free monoid containing no nonzero idempotents, and let $G$ be the set of invertible elements of $S$. Assume that $f$ is a unit of $R[X ; S]$, that $f_{1}$ is the 
G-component of $f$, and that $f_{2}$ is the $(S-G)$-component of $f$. Then $f$ has finite order if and only if $f_{1}$ has finite order and $f_{2}$ has finite additive order.

Proof. It follows from Theorem 3.2 that $f_{1}$ is a unit of $R[X ; S]$ and that $f_{2}$ is nilpotent. Therefore Theorem 4.1 implies that $f$ has finite order if $f_{1}$ has finite order and $f_{2}$ has finite additive order. Conversely, if $f$ has finite order $t$, then the G-component of $f^{t}=1$ is $1=f_{1}^{t}$. Thus $f_{1}$ has finite order. Since $f_{2}$ is nilpotent and $f=f_{1}+f_{2}$ has finite order, $f_{2}$ has finite additive order by Theorem 4.2.

The condition in Theorem 4.4 that $f_{2}$ should have finite order is completely determined by the coefficients of $f_{2}$ (which are coefficients of $f$ ), but we seek a more definitive criterion for determining whether $f_{1}$ is a unit of $R[X ; G]$ of finite order. The desired result is stated in Theorem 4.6, which requires one preliminary result.

Proposition 4.5. Assume that the polynomial $f=f_{0}+f_{1} X+$ $\cdots+f_{j} X^{j}+\cdots+f_{n} X^{n} \in R[X]$ is such that $f_{j}$ is a unit of $R$, while $f_{i}$ is nilpotent for each $i \neq j$. If there exists a positive integer $h$ such that $f^{h}=X^{j h}$, then $f_{j}$ has finite order.

Proof. Let $A$ be the ideal of $R$ generated by $\left\{f_{i} \mid i \neq j\right\}$. We claim that for any $m>0$, there exists a positive integer $k$ such that $k A^{m}=(0)$. Since $A$ is nilpotent, we can take $k=1$ for all sufficiently large $m$. Assume the the integer $t \geqq 1$ is such that the claim is true for all $m>t$. We prove the claim for $t$. We can choose $k$ so that $k A^{m}=(0)$ for each $m>t$. For some $h$ we have $f^{h}=X^{h j}$, and hence $k f^{h}=k X^{h j}$. For $i \neq j$, the coefficient of $X^{(h-1) j+i}$ in $k f^{h}$ is $0=k\left[\left(\begin{array}{l}h \\ 1\end{array}\right) f_{j}^{h-1} f_{i}+u\right]$, where $u$ is in $A^{2}$. This statement is true because the coefficient of $X^{(h-1) j+i}$ is a sum of products of $h$ coefficients $f_{i_{1}}, \cdots, f_{i_{h}}$ of $f$, where $i_{1}+i_{2}+\cdots+i_{h}=(h-1) j+i$; if at most one of the elements $f_{i_{c}}$ is in $A$, then $h-1$ of them must be $f_{j}$ and the other must be $f_{i}$. Therefore

$$
\begin{aligned}
& k h f_{j}^{h-1} f_{i}=-k u \\
& k h f_{j}^{h-1} f_{i} A^{t-1}=-k u A^{t-1} \cong k A^{2} A^{t-1}=k A^{t+1}=(0) .
\end{aligned}
$$

Since $f_{j}$ is a unit of $R$, it follows that $\operatorname{kh} f_{i} A^{t-1}=(0)$ for each $i \neq j$, and consequenlty $k h A^{t}=(0)$, thereby establishing the claim. The claim implies that there exists a positive integer $K$ such that $K A=(0)$. We proceed to show that $f_{j}$ has finite order.

In the ring $R\left[X, X^{-1}\right]$, we have

$$
f / X^{j}=f_{j}+\left[\left(f_{0} / X^{j}\right)+\cdots+\left(f_{j-1} / X\right)+f_{j+1} X+\cdots+f_{n} X^{n-j}\right],
$$


where $\left(f / X^{j}\right)^{h}=X^{j h} / X^{j h}=1$. Therefore $f / X^{j}$ is a unit of finite order, where $f_{j}$ is a unit and $\left[\left(f_{0} / X^{j}\right)+\cdots+f_{n} X^{n-j}\right]$ is nilpotent of finite additive order (since $K A=(0)$ ). Applying Theorem 4.1, we conclude that $f_{j}$ has finite order, and this completes the proof of Proposition 4.5 .

THEOREM 4.6. Let $G$ be a torsion-free abelian group and let $f=a_{1} X^{s_{1}}+\cdots+a_{m} X^{s_{m}}+a_{m+1} X^{s_{m+1}}+\cdots+a_{n} X^{s_{n}}$ be a unit of $R[X ; G]$, where the labelling is such that $a_{i}$ is not nilpotent for $1 \leqq i \leqq m$ and $a_{i}$ is nilpotent for $m+1 \leqq i \leqq n$. The following conditions are equivalent.

(1) The unit $f$ has finite order.

(2) $m=1, s_{1}=0, a_{1}$ is a unit of $R$ of finite order, and the element $a_{m+1} X^{s_{m+1}}+\cdots+a_{n} X^{s_{n}}$ has finite additive order.

Proof. It is clear that (2) implies (1). To prove the converse, let $\alpha=a_{1} X^{s_{1}}+\cdots+a_{m} X^{s_{m}}$ and let $\beta=a_{m+1} X^{s_{m+1}}+\cdots+a_{n} X^{s_{n}}$. Since $G$ is torsion-free, it admits a total order $<$, and we assume without loss of generality that the labelling is such that $s_{1}<s_{2}<$ $\cdots<s_{m}$. Let $N$ be the nilradical of $R$ and let $\varphi$ be the canonical homomorphism of $R[X ; G]$ onto $(R / N)[X ; G]$. If $f$ has order $t$, then

$$
\begin{aligned}
1= & {[\varphi(f)]^{t}=\left[\varphi\left(a_{1}\right) X^{s_{1}}+\cdots+\varphi\left(a_{m}\right) X^{s_{m}}\right]^{t} } \\
= & {\left[\varphi\left(a_{1}\right)\right]^{t} X^{t s_{1}}+t\left[\left(\varphi\left(a_{1}\right)\right)^{t-1} \varphi\left(a_{2}\right)\right] X^{(t-1) s_{1}+s_{2}}+\cdots } \\
& +t\left[\varphi\left(a_{m-1}\right)\left(\varphi\left(a_{m}\right)\right)^{t-1}\right] X^{s_{m-1}+(t-1) s_{m}}+\left[\varphi\left(a_{m}\right)\right]^{t} X^{t s_{m}},
\end{aligned}
$$

where $t s_{1}<(t-1) s_{1}+s_{2}<\cdots<t s_{m}$ and where $\left(\varphi\left(a_{1}\right)\right)^{t} \neq 0$ and $\left(\varphi\left(a_{m}\right)\right)^{t} \neq 0$. If $m>1$, it follows that $[\varphi(f)]^{t}$ is not a monomial. Hence $m=1, t s_{1}=0$, and $\varphi\left(a_{1}\right)$ is a unit of $R / N$. Since $G$ is torsionfree, it follows that $s_{1}=0$. Moreover, $a_{1}$ is a unit of $R$. To complete the proof, we must show that $a_{1}$ has finite order and that $\beta$ has finite additive order; by Theorem 4.1 and Corollary 4.2, either of these statements follows from the other, and hence we prove only that $a_{1}$ has finite order.

Let $G_{0}$ be the subgroup of $G$ generated by $\operatorname{Supp}(f) \cup \operatorname{Supp}\left(f^{-1}\right)$. Then $f$ is a unit of the group ring $R\left[X ; G_{0}\right]$, and $G_{0}$ is finitely generated. Replacing $G$ by $G_{0}$, we may assume without loss of generality that $G=\boldsymbol{Z} \oplus \cdots \oplus \boldsymbol{Z}$ is a direct sum of $k$ copies of $\boldsymbol{Z}$ [5, Theorem 16]. Hence $R[X ; G]$ is, to within isomorphism, the quotient ring $R\left[X_{1}^{ \pm 1}, \cdots, X_{k}^{ \pm 1}\right]$ of the polynomial ring $R\left[X_{1}, \cdots, X_{k}\right]$ in $k$ indeterminates over $R$. Considering $R[X ; G]$ to be $R\left[X_{1}^{ \pm 1}, \cdots, X_{k}^{ \pm 1}\right]$, we show that the constant term of $f$ is of finite order by induction on $k$. If $k=1$, we can assume that

$$
f=b_{-r} X^{-r}+\cdots+b_{-1} X^{-1}+b_{0}+b_{1} X+\cdots+b_{s} X^{s},
$$


where $b_{0}$ is a unit of $R$ and each other coefficient of $f$ is nilpotent. If $f$ has order $t$, then $h=X^{r} f \in R[X]$ and $h^{t}=X^{r t}$. By Proposition $4.5, b_{0}$ is of finite order. Therefore the desired conclusion holds if $k=1$.

If the conclusion is assumed for $G$ of rank $r$, then in the case of a group of rank $r+1$, we consider $R\left[X_{1}^{ \pm 1}, \cdots, X_{r+1}^{ \pm 1}\right]$ as $R\left[X_{1}^{ \pm 1}, \cdots, X_{r}^{ \pm 1}\right]\left[X_{r+1}^{ \pm 1}\right]$. Writing $f$ as a polynomial in $X_{r+1}^{ \pm 1}$ with coefficients in $R\left[X_{1}^{ \pm 1}, \cdots, X_{r}^{ \pm 1}\right]$, the coefficient $h$ of $X_{r+1}^{0}$ is a unit of $R\left[X_{1}^{ \pm 1}, \cdots, X_{r}^{ \pm 1}\right]$ and all other coefficients are nilpotent. The case where $G$ is of rank 1 implies that $h$ is a unit of $R\left[X_{1}^{ \pm 1}, \cdots, X_{r}^{ \pm 1}\right]$ of finite order. But $h$ is such that its coefficient $b_{0}$ of $X_{1}^{0} X_{2}^{0} \cdots X_{r}^{0}$ is a unit of $R$, while all other coefficients are nilpotent. It then follows from the induction hypothesis that $b_{0}$ has finite order, and this completes the proof of Theorem 4.6.

Combining Theorems 4.4 and 4.6, we obtain another description of the units of $R[X ; S]$ of finite order.

THEOREM 4.7. Assume that $(S,+)$ is a torsion-free monoid containing no nonzero idempotents. An element $f$ of $R[X ; S]$ is a unit of finite order if and only if $0 \in \operatorname{Supp}(f)$, the coefficient of $X^{0}$ in $f$ is a unit of finite order, and each other coefficient of $f$ is a nilpotent element of finite additive order.

For a ring $T$, let $U(T)$ denote the group of units of $T$. Theorems 3.2 and 4.7 yield information concerning the structure of the group $U(R[X ; S])$ and its torsion subgroup in the case where $S$ is torsionfree and contains no nonzero idempotents. To wit, let $G$ be the group of invertible elements of $S$, let $N$ be the nilradical of $R$, and let $C$ be the ideal of $R$ consisting of nilpotent elements of finite additive order. Theorem 3.2 implies that $U(R[X ; S])$ is the direct product of $U(R[X ; G])$ and the group $1+N[X ; S-G]$, while Theorem 4.7 shows that the torsion subgroup of $U(R[X ; S])$ is the direct product of the torsion subgroup of $U(R)$ and the group $1+C[X ; S-\{0\}]$.

\section{REFERENCES}

1. A. H. Clifford and G. B. Preston, The Algebraic Theory of Semigroups, Amer. Math. Soc., Providence, R. I., 1961.

2. R. Gilmer and T. Parker, Divisibility properties in commutative semigroup rings. Michigan Math. J., 21 (1974), 65-86.

3. R. Gilmer and M. L. Teply, Idempotents of commutative semigroup rings, Houston J. Math., 3 (1977), 369-385.

4. G. Higman, The units of group rings, Proc. London Math. Soc., 46 (1940), 231-248.

5. I. Kaplansky, Infinite Abelian Groups, Univ. of Michigan Press, Ann Arbor, Mich., 1969.

6. D. G. Northcott, Lessons on Rings, Modules, and Multiplicities, Cambridge Univ. 
Press, London, 1968.

7. T. Parker and R. Gilmer, Nilpotent elements of commutative semigroup rings, Michigan Math. J., 22 (1975), 97-108.

8. S. K. Sehgal, On the isomorphism of integral group rings. I., Canad. J. Math., 22 (1969), 410-413.

9. - Units of commutative integral group rings, Math. J. Okayama Univ., 14 (1970), 135-138.

10. E. Snapper, Completely primary rings. I., Annals of Math., 52 (1950), 666-693.

Received November 29, 1978.

Florida State University

TALLAHASSE, FL 32306

AND

University of TeXas at AUSTIN

AUSTIN, TX 78712 


\section{PACIFIC JOURNAL OF MATHEMATICS}

\section{EDITORS}

DONALD BABBITT (Managing Editor)

University of California

Los Angeles, California 90024

HUgo RossI

University of Utah

Salt Lake City, UT 84112

C. C. MOORE and ANDREW OGG

University of California

Berkeley, CA 94720

\section{J. DUGUNDJI}

Department of Mathematics University of Southern California Los Angeles, California 90007

R. Finn and J. Milgram Stanford University Stanford, California 94305

\section{ASSOCIATE EDITORS}

E. F. BECKENBACH

B. H. NEUMANN

F. WOLF

K. YoshidA

\section{SUPPORTING INSTITUTIONS}

UNIVERSITY OF BRITISH COLUMBIA CALIFORNIA INSTITUTE OF TECHNOLOGY UNIVERSITY OF CALIFORNIA MONTANA STATE UNIVERSITY UNIVERSITY OF NEVADA, RENO NEW MEXICO STATE UNIVERSITY OREGON STATE UNIVERSITY UNIVERSITY OF OREGON
UNIVERSITY OF SOUTHERN CALIFORNIA STANFORD UNIVERSITY UNIVERSITY OF HAWAII UNIVERSITY OF TOKYO UNIVERSITY OF UTAH WASHINGTON STATE UNIVERSITY UNIVERSITY OF WASHINGTON 


\section{Pacific Journal of Mathematics}

Vol. 85, No. $1 \quad$ September, 1979

Ralph Alexander, Metric averaging in Euclidean and Hilbert spaces...... 1

B. Aupetit, Une généralisation du théorème de Gleason-Kahane-Żelazko

pour les algèbres de Banach..............................

Lung O. Chung, Jiang Luh and Anthony N. Richoux, Derivations and

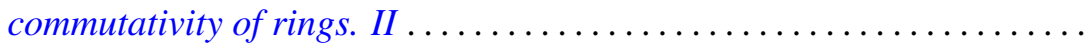

Lynn Harry Erbe, Integral comparison theorems for third order linear

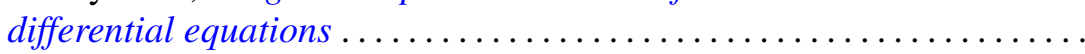

Robert William Gilmer, Jr. and Raymond Heitmann, The group of units of a

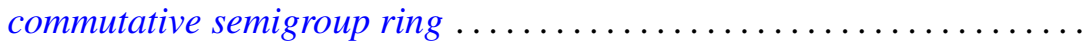

George Grätzer, Craig Robert Platt and George William Sands, Embedding lattices into lattices of ideals ...........................

Raymond D. Holmes and Anthony Charles Thompson, $n$-dimensional area and content in Minkowski spaces ....................... 77

Harvey Bayard Keynes and M. Sears, Modelling expansion in real flows....

Taw Pin Lim, Some classes of rings with involution satisfying the standard

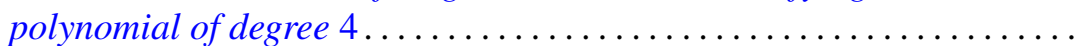

Garr S. Lystad and Albert Robert Stralka, Semilattices having bialgebraic congruence lattices ................................... 131

Theodore Mitchell, Invariant means and analytic actions . . . . . . . . . 145

Daniel M. Oberlin, Translation-invariant operators of weak type ........ 155

Raymond Moos Redheffer and Wolfgang V. Walter, Inequalities involving

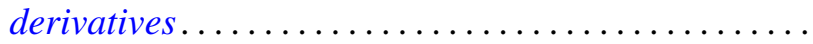

Eric Schechter, Stability conditions for nonlinear products and semigroups ................................

Jan Søreng, Symmetric shift registers ........................ 201

Toshiji Terada, On spaces whose Stone-Čech compactification is $\mathrm{Oz} \ldots \ldots .231$

Richard Vrem, Harmonic analysis on compact hypergroups ... 